\title{
TEE-based Privacy-Preserve in Collaborative Traffic Policy Compilation for Programmable Devices
}

\author{
Aris Cahyadi Risdianto \\ aris@comp.nus.edu.sg \\ National University of Singapore (NUS) \\ Singapore
}

\author{
Ee-Chien Chang \\ changec@comp.nus.edu.sg \\ National University of Singapore (NUS) \\ Singapore
}

\begin{abstract}
Maintaining the integrity of network devices policy across a different organization is very challenging since the devices are shared for multiple traffic forwarding purposes, including public Internet access. An organization's administrator can put unnecessary (i.e., wrong) policy that may leak the private traffic between the organizations to a public network. It can be avoided by exchanging the network traffic policy between the organizations but keeping the confidentiality of the policies among them (i.e., to avoid honest-butcurious adversary) is very challenging. Furthermore, there is also no guarantee that the policy is properly enforced into the network device. An administrator can intentionally put malicious policies that allow the attacker to enter the organization's network (i.e., malicious adversary). This paper proposed a cross-organization network traffic policy compilation that preserves the policy privacy and ensures its enforcement to the network devices. It utilizes a trusted execution environment (TEE) to compile the high-level traffic policies into low-level rules for the programmable network device. Then, the rules are easily pushed and optimized by using hardware programming abstraction.
\end{abstract}

\section{CCS CONCEPTS}

• Networks $\rightarrow$ Network security; Programmable networks; • Security and privacy $\rightarrow$ Privacy-preserving protocols.

\section{KEYWORDS}

Collaborative processing; privacy preserving; programmable device; traffic policy; trusted computing

\section{ACM Reference Format:}

Aris Cahyadi Risdianto and Ee-Chien Chang. 2021. TEE-based PrivacyPreserve in Collaborative Traffic Policy Compilation for Programmable Devices. In Proceedings of the 2021 ACM International Workshop on Software Defined Networks \& Network Function Virtualization Security (SDN-NFV Sec'21), April 28, 2021, Virtual Event, USA. ACM, New York, NY, USA, 4 pages. https://doi.org/10.1145/3445968.3452091

Permission to make digital or hard copies of all or part of this work for personal or classroom use is granted without fee provided that copies are not made or distributed for profit or commercial advantage and that copies bear this notice and the full citation on the first page. Copyrights for components of this work owned by others than ACM must be honored. Abstracting with credit is permitted. To copy otherwise, or republish, to post on servers or to redistribute to lists, requires prior specific permission and/or a fee. Request permissions from permissions@acm.org.

SDN-NFV Sec'21, April 28, 2021, Virtual Event, USA

(c) 2021 Association for Computing Machinery.

ACM ISBN 978-1-4503-8318-9/21/04 . \$ \$15.00

https://doi.org/10.1145/3445968.3452091

\section{INTRODUCTION}

Most of the organization network devices (e.g., router or firewall) are used simultaneously for public traffic forwarding (i.e., Internet access) and private traffic forwarding across trusted organizations (i.e., virtual private network or VPN). Private traffic can be leaked to the public domain due to a policy inconsistency issue in the network devices. So, the organization's administrators need to ensure there are no such problematic policies in their devices. It is possible if they exchange (i.e., share) the complete traffic policies amongst them. Unfortunately, that is not an easy task and against the security best practices. Each organization's traffic policy (e.g., where to send and what kind of traffic) should not be exchanged amongst them, even though they have no intention to attack each other. It is required for each administrator to exchange traffic policies without revealing them to preserve privacy. They also need to ensure proper policy enforcement by the administrators without modification to avoid an attacker entering the organization. So, the high-level traffic policies between organizations need to be exchanged trustfully (i.e., securely) and compiled into low-level rules in the network devices. A trusted execution environment (TEE) can be used for a trusted policy-to-rule compilation mechanism. It also includes verifying the policy enforcement into the device rule by continuously comparing the newly generated policy with the existing rules to avoid conflicting and overlapping. This task is simpler to achieve with a programmable network paradigm that gives robust methods to query and push rules at the runtime. It also allows us to optimize (e.g., aggregate) the device's rules. The overall concept is shown in Fig. 1.

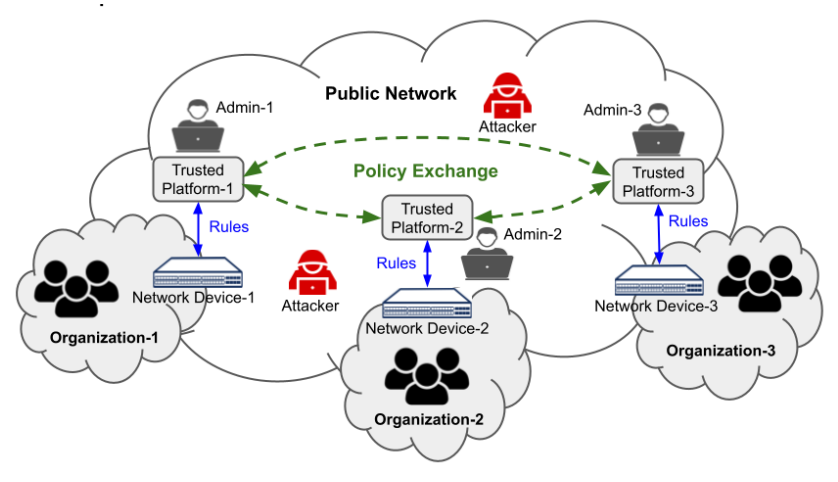

Figure 1: The overall concept. 
TEE is designed to fulfill the secure and trusted computation requirement by leveraging trusted hardware such as Intel's Software Guard Extensions (SGX). This latest hardware design protects selected code and data from disclosure or modification by partition the computation services into hardened enclaves to increase data confidentiality and integrity when the computation is being performed on it [6]. One of the essential features of the TEE, called attestation, enables local attestation between enclaves or remote attestation by a "relying party" to help ensure the computation process or the data are not compromised. A portion of computational software can be loaded into an enclave that sent the report to the remote computation users to validate whether an authentic CPU generated the report. Thus, it also includes an instruction for generating a CPU/enclave specific "sealing key" that can be used to safely store and retrieve sensitive information that needs to be stored or protected outside the enclave [12]. So, the TEE can be used as a trusted platform for a policy-to-rule compilation of network traffic across organizations that keep the policy confidential and check its enforcement in network devices.

$\mathrm{P} 4$ is a high-level language to define packet processing by the data plane of a programmable forwarding element such as a hardware/software switch, network interface card, router, or network appliance [5]. So, the target element can be programmed into specific network devices such as a firewall by specifying P4-based firewall functions that control packet-forwarding functions in the data plane. The rules can be easily configurable to update functionalities through data plane runtime on the fly [7]. It means that the device's rules are easily modified based on the compiled network traffic policy from the trusted computation platform almost in real-time.

This paper discusses how to utilize TEE for traffic policies compilation across organizations while still preserving privacy and enforcing it into rules in the programmable device. The organization of the paper as follows. It starts with Section 1 as an introduction and Section 2 as our proposed solution. Then, Section 3 discusses the implementation and Section 4 shows the verification. It concludes with Section 5 and 6 for discussion and conclusion.

\section{PRIVACY PRESERVE TRAFFIC POLICY}

\subsection{Motivation}

As mentioned in these works [14][15], there are needs for administrators across organizations to ensure policy consistency in their network devices. So, they need to establish additional centralized policy control or exchange the policies amongst them. Centralized control needs third-party authority management and adds more delay in policy processing. Policy exchange (i.e., sharing) is easier but it is not a good security practice. Even though they have no intention to "exploit" the shared policies, but learning other organization policies is exposing its privacy (i.e., honest-but-curious adversary). Let assume if they already have a secure mechanism to exchange the policies, but ensuring the policy enforcement into a network device is also very important. One organization administrator can inject a malicious policy that may violate another organization's policy or existing device rules. That policy may also allow the attacker from the public domain to enter respective organizations and to do malicious activities (i.e., malicious adversary).

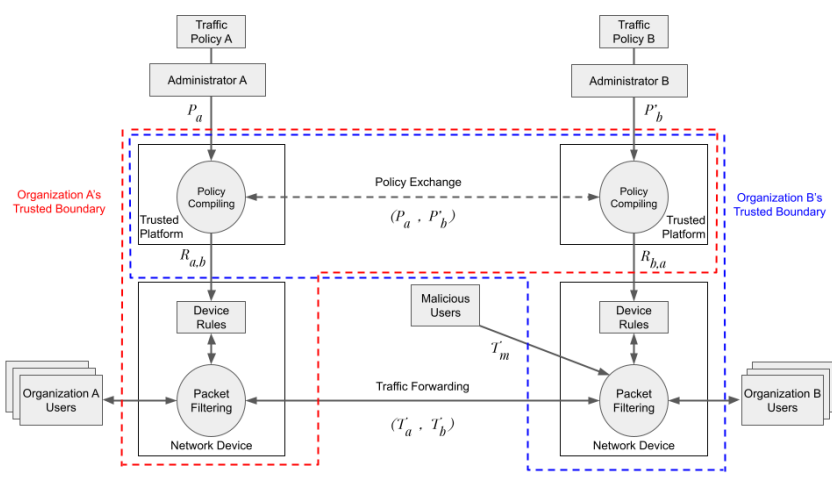

Figure 2: Threat Model.

\subsection{Threat Model and Assumption}

Threat Model. As shown in Fig. 2, organization A and B want to secure private traffic between their users. Administrator A and B need to exchange the policy $P_{a}$ and $P_{b}$ to ensure the policy consistency before enforcing rules $R_{a, b}$ and $R_{b, a}$ to the network device $\mathrm{A}$ and $\mathrm{B}$, respectively. In this case, Administrator B can reveal $P_{a}$ that may not only be used to control the private traffic forwarding $T_{a}$ and $T_{b}$ between organization A and B. Although Administrator B has no intention to leak or exploit $P_{a}$ but $P_{a}$ privacy is not preserved. It is considered as a honest-but-curious adversary model. However, Organizations A and B can establish a secured policy exchange mechanism, but Administrators A and B still need to ensure that the policy only allows traffic between their organization. Unfortunately, Administrator B can intentionally push a policy that contains a malicious policy $P_{b}^{\prime}$ to his trusted platform that may allow a malicious user to send malicious traffic $T_{m}$ to organization B. The malicious user may attack organization B's user directly or even organization A's user via organization B's infrastructure. So, it can be considered as a malicious adversary model.

Assumptions. Several conditions are required to solve those specific adversary models. First, We assume that a secure communication channel (e.g., secure socket layer or SSL) can be established between the organization's trusted platform to protect policies exchange between platforms. It means the received input policy from each trusted platform is always valid. Second, the administrator unable to access the platform to interrupt the policy compilation process. They can only insert the input policy to the platform and check the generated rules in the devices. Third, the distance between the trusted platform and the programmable network device is very near, or the link between them is isolated. So, only the trusted platform can push the rule into devices. The administrators are unable to push rules directly to the device to avoid direct malicious rule insertion.

\subsection{Policy Confidentiality and Integrity}

The new mechanism should be designed by considering a simple mechanism to address those requirements. It should avoid complex processing, use a trusted platform to avoid fake/malicious program injection, and also consider policy enforcement into device rule. 
By leveraging a trusted platform, the privacy-preserve policy compilation should be easier to achieve. The input policies only can be exchanged and trustfully compiled into device rules inside the trusted platform. Then, generated rules should be consistent without any conflicting or overlapping rules, and irreversible that is impossible to derive input policy from it. In summary, our contributions are:

- Propose a method to keep the confidentiality of traffic policies from a honest-but-curious adversary where highlevel policies are securely exchanged and trustfully compiled into low-level network device rules.

- Propose a method to provide a significant integrity level of the rules because it must be only compiled from the matched policies from both organizations and existing rules.

\section{TRUSTED POLICY-TO-RULE COMPILATION}

\subsection{Policy Input}

The trusted policy-to-rule compilation platform consists of distributed TEE hardware across multiple organizations. It allows the organization's administrators to push their traffic policy, but it prevents them from accessing the hardware directly. TEE's enclave can be created by the administrator using a set of instructions by untrusted third-party software before it can be utilized to run the policy compilation software inside [6]. For ensuring the administrator can communicate with that trusted software, the attestation process is required to provide a cryptographic signature that certifies the hash of that enclave to the administrator.

Allowing multiple administrators to share the policies requires a remote attestation between enclaves. It verifies the shared policies from local enclave to remote enclave or vice versa are met the security model by using asymmetric cryptography. Before enclaves can share the policy input, enclaves need to establish a secure communication channel (i.e., SSL) between them. Importantly one SSL session only allows one-time policy input exchange to avoid a "recursion" attack of the policy. For example, when Administrator A share Policy A, the enclave A establish SSL session to enclave B to get Policy B. Then, enclave A compiles both policies into rules and destroys both policies' information. So, there is no possibility that Administrator A can continuously inject multiple combinations of Policy A with the same Policy B from the previous process to predict Policy B based on generated rules.

\subsection{Policy Modelling and Representation}

In this work, a policy is defined as an ordered criterion (i.e., set of filter criteria) with defined actions to be performed on packets that satisfy the criterion. The criterion consists of different filtering criteria for network packet fields (e.g., protocol type, source/destination IP address, and source/destination port) and different actions (e.g., accept or reject). Each network packet field could be a single value or a range of values (i.e., wildcard) that can make the criterion overlap/conflict with each other. So, modeling the policies and criterion relations is crucial to check and validate the traffic policy input from different organizations.

Several simple methods of policy modeling and representation has been proposed by [1][9]. Because we implement the policy compilation in a trusted platform, so we can use it easily without a complex method as proposed by [3]. First, we use set diagrams for traffic policies with policy criterion as the component of the set. Secondly, we also use the binary representation for the policy criterion, especially for port number and IP address criteria, because it can be used to detect overlapping between policy criterion and invalid criteria.

\subsection{Policy Checking and Matching}

As shown in Fig 3, there are several processes in compiling policy from two different organizations into network device rule. Interpolicy Checking utilizes a set diagram representation for the policy criterion and applies the "exact match" operation between two organization's policies to find the same criterion, as mentioned in this work. Intra-policy Checking is required to prevent the traffic leakage to the public network due to an "overlapping" or "shadowing" match of the criteria (e.g., IP address or port number) between policy criterion in a single organization. By leveraging available binary search algorithms [13], we use the longest match prefix (LPM) to find the overlapping IP address in a Binary Trie representation. Additionally, IP address with "any" values (i.e., 0.0.0.0/0) can cause major overlapping and leaking of IP addresses. So, we consider any criterion with this "invalid" IP address will not be added as a valid policy criterion.

\subsection{Policy-to-rule Generation}

The policy of modern networks is not easy to be described and decoupled. Because it is usually defined at the low level of rules on the underlying hardware, and it does not support dynamic policy implementation. Our proposed policy compilation needs to compile inputs from both valid policy criterion and existing rules in the network device. We adopt the $\mathrm{P} 4$ data structure serialization (i.e., protobuf) and $\mathrm{P} 4$ runtime system to query, install, uninstall the low-level packet processing rules on the P4 network device. Our policy compiler can extract the protobuf message and match it with the valid policy before optimizing it to generate "compiled" rules and push it back to the device.

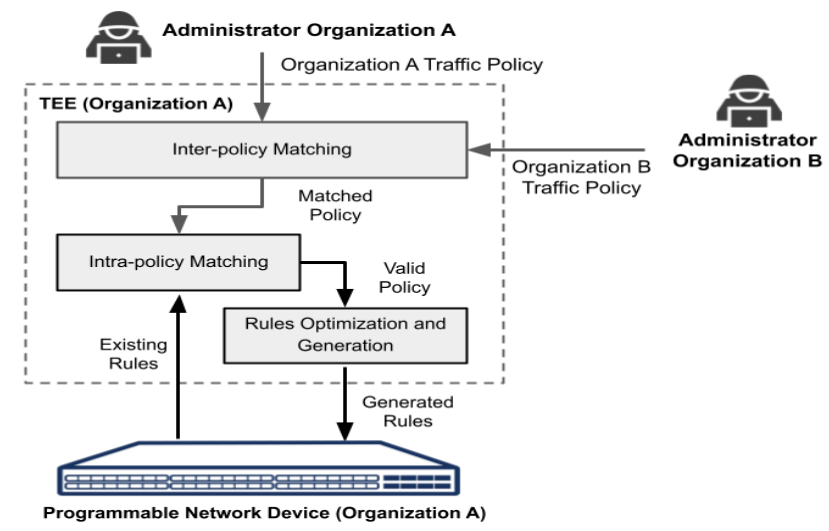

Figure 3: Policy-to-Rule Compilation. 
Table 1: Example Policy and Rules

\begin{tabular}{|c|c|c|c|c|c|c|}
\hline No & Prot & SrcPort & DestPort & SrcIP & DestIP & Action \\
\hline \multicolumn{7}{|c|}{ Example Traffic Policy of Organization A } \\
\hline 1 & tсp & any & 22 & $10.1 .1 .10 / 32$ & 172.16.1.10/32 & accept \\
\hline 2 & tсp & 22 & any & 172.16.1.10/32 & 10.1.1.10/32 & accept \\
\hline 3 & tcp & any & 22 & $10.1 \cdot 1 \cdot 10 / 16$ & $172.16 .1 .10 / 32$ & accept \\
\hline \multicolumn{7}{|c|}{ Example Traffic Policy of Organization B } \\
\hline 1 & tcp & any & 22 & 10.1.1.10/32 & 172.16.1.10/32 & accept \\
\hline 2 & tcp & 22 & any & 172.16.1.10/32 & 10.1.1.10/32 & accept \\
\hline 3 & tcp & any & 22 & any & 172.16.1.10/32 & accept \\
\hline 4 & tcp & 80 & any & 172.16.1.10/32 & any & accept \\
\hline \multicolumn{7}{|c|}{ Example "Existing" Rules for Device A } \\
\hline 1 & tcp & any & 22 & 10.1.1.10/32 & 172.16.1.8/32 & accept \\
\hline 2 & tcp & any & 22 & $10.1 .1 .10 / 16$ & 172.16.1.9/32 & accept \\
\hline 3 & tcp & any & 22 & 10.1.1.10/16 & 172.16.1.11/32 & accept \\
\hline 4 & tcp & 22 & any & $172.16 .1 .9 / 32$ & $10.1 .1 .10 / 32$ & accept \\
\hline \multicolumn{7}{|c|}{ Example "Compiled" Rules for Device A } \\
\hline 1 & tcp & any & 22 & 10.1.1.10/32 & $172.16 .1 .8 / 30$ & accept \\
\hline 2 & tcp & 22 & any & 172.16.1.9/32 & $10.1 .1 .10 / 32$ & accept \\
\hline
\end{tabular}

\section{EXPERIMENT}

We implement all the components in a Python program with several libraries (e.g., math, $\mathrm{P} 4$ runtime, and protobuf) as initial implementation. We use two organization policies and one existing P4 device rules as input for our initial program verification. Table 1 shows the example of input policies from Organization A and Organization B, Device A's existing rules taken from $\mathrm{P} 4$ runtime protobuf message [8], and also the compiled rules for Device A.

Thus, we create several combinations of Policy B to verify the accuracy and efficiency of our program. We mix the Policy B entries from the previous table with multiple policy rules data with randomly generated criteria [16]. Our experiment results show that our program can successfully compile policies with different numbers of additional policy entries for Policy B from 10 to 1000 .

\section{DISCUSSION AND RELATED WORK}

There are many "simple" traffic policy processing which focused on modelling and analyzing a relation between policy entries [1][9][10] [11][18]. Especially for these works [1][10], they can detect a conflict and anomaly between policy entries. Unfortunately, most of them are not preserve the privacy of the policies because it is used only for single organization policy processing. So, several works improve it by providing privacy preserve mechanism such as [3][4][14][15] for cross-domain policy processing. Especially, Alex and Fei [14][15] proposed very interesting approaches for collaborative policy processing methods in VPN. It allows roaming users in the outside network to access some resources that are only accessible from their organization network without exposing the policy between outside and organization network policies. They proposed several "complex" mechanisms to fulfill their needs, including oblivious comparison with Xhash, FDD (Firewall Decision Diagram) construction, and prefix numericalizations.

Our proposed mechanism tried to improve those "complex" works [1][10] by utilizing trusted platform to compile policies, so that any other "simple" works [1][9][10][11][18] also can be utilized. Then, our policy compiler [16] able to compile the valid policy input and existing device rules by leveraging wide-available functions. Even though, it still runs outside TEE but it should be easily executed inside the enclave by natively running it using SCONE [2] or Graphene-SGX [17]. Alternatively, the code can be migrated into SGX's SDK-supported language (e.g., $\mathrm{C} / \mathrm{C}++$ and Rush).

\section{CONCLUSION}

This paper shows that a TEE can be used to collaboratively compile the high-level network traffic policies from multiple organizations into low-level rules of their programmable network device. The proposed policy compiler works with a different number of policy entries, and it can generate the "expected" rules for the devices. Currently, we are in progress to implement it in SGX and force the rules into Tofino-based switches.

\section{ACKNOWLEDGMENTS}

This research is supported by the National Research Foundation, Prime Ministers Office, Singapore under its National Cybersecurity R\&D Program (Award No. NRF-NCL-P2-0001) and administered by the National Cybersecurity R\&D Directorate.

\section{REFERENCES}

[1] Ehab Al-Shaer, Hazem Hamed, Raouf Boutaba, and Masum Hasan. 2005. Conflict classification and analysis of distributed firewall policies. IEEE journal on selected areas in communications 23, 10 (2005), 2069-2084.

[2] Sergei Arnautov, Bohdan Trach, Franz Gregor, Thomas Knauth, Andre Martin, Christian Priebe, Joshua Lind, Divya Muthukumaran, Dan O'keeffe, Mark L Stillwell, et al. 2016. \{SCONE\}: Secure linux containers with intel \{SGX\}. In 12th $\{$ USENIX\} Symposium on Operating Systems Design and Implementation (\{OSDI\} 16). 689-703.

[3] Fei Chen, Bezawada Bruhadeshwar, and Alex X Liu. 2012. Cross-domain privacypreserving cooperative firewall optimization. IEEE/ACM transactions on Networking 21, 3 (2012), 857-868.

[4] Jerry Cheng, Hao Yang, Starsky HY Wong, Petros Zerfos, and Songwu Lu. 2007. Design and implementation of cross-domain cooperative firewall. In 2007 IEEE International Conference on Network Protocols. IEEE, 284-293.

[5] P4 Language Consortium et al. [n.d.]. P4 language and related specifications. URL: https://p4.org/specs ([n. d.]).

[6] Victor Costan and Srinivas Devadas. 2016. Intel SGX Explained. IACR Cryptol. ePrint Arch. 2016, 86 (2016), 1-118.

[7] Rakesh Datta, Sean Choi, Anurag Chowdhary, and Younghee Park. 2018. P4guard: Designing p4 based firewall. In MILCOM 2018-2018 IEEE Military Communications Conference (MILCOM). IEEE, 1-6.

[8] Google Developers. [n.d.]. Protocol Buffers. https://developers.google.com/protocol-buffers ([n.d.])

[9] Scott Hazelhurst. 2000. Algorithms for analysing firewall and router access lists. arXiv preprint cs/0008006 (2000).

[10] Hongxin Hu, Gail-Joon Ahn, and Ketan Kulkarni. 2012. Detecting and resolving firewall policy anomalies. IEEE Transactions on dependable and secure computing 9, 3 (2012), 318-331.

[11] Alan Jeffrey and Taghrid Samak. 2009. Model checking firewall policy configurations. In 2009 IEEE International Symposium on Policies for Distributed Systems and Networks. IEEE, 60-67.

[12] Simon Johnson, Vinnie Scarlata, Carlos Rozas, Ernie Brickell, and Frank Mckeen. 2016. Intel ${ }^{\circledR}$ software guard extensions: Epid provisioning and attestation services. White Paper 1, 1-10 (2016), 119.

[13] H. Lim and N. Lee. 2012. Survey and Proposal on Binary Search Algorithms for Longest Prefix Match. IEEE Communications Surveys Tutorials 14, 3 (2012), 681-697. https://doi.org/10.1109/SURV.2011.061411.00095

[14] Alex X Liu and Fei Chen. 2008. Collaborative enforcement of firewall policies in virtual private networks. In Proceedings of the twenty-seventh ACM symposium on Principles of distributed computing. 95-104.

[15] Alex X Liu and Fei Chen. 2010. Privacy preserving collaborative enforcement of firewall policies in virtual private networks. IEEE Transactions on Parallel and Distributed Systems 22, 5 (2010), 887-895.

[16] Aris C. Risdianto. [n.d.]. Collaborative Policy Compiler.

$U R L:$ https://github.com/ariscahyadi/collaborative-policy-compiler ([n. d.]).

[17] Chia-Che Tsai, Donald E Porter, and Mona Vij. 2017. Graphene-sgx: A practical library $\{$ OS $\}$ for unmodified applications on $\{$ SGX $\}$. In $2017\{$ USENIX\} Annual Technical Conference ( $\{$ USENIX $\}\{A T C\}$ 17). 645-658.

[18] Lihua Yuan, Hao Chen, Jianning Mai, Chen-Nee Chuah, Zhendong Su, and Prasant Mohapatra. 2006. Fireman: A toolkit for firewall modeling and analysis. In 2006 IEEE Symposium on Security and Privacy (S\&P'06). IEEE, 15-pp. 